\title{
Letter to the Editor regarding "Identification of the oleic acid ethanolamide (OEA) isomer cis-vaccenic acid ethanolamide (VEA) as a highly abundant 18:1 fatty acid ethanolamide in blood plasma from rats and humans"
}

\author{
Dimitrios Tsikas ${ }^{1}$
}

Received: 13 January 2017 / Accepted: 26 January 2017 / Published online: 6 February 2017

(C) Springer-Verlag Berlin Heidelberg 2017

Dear Editors, dear authors:

I read with great interest the recent research paper by Röhrig et al. in Anal Bioanal Chem 2016 [1], which reported on the unequivocal identification of cis-vaccenic acid ethanolamide (VEA) in blood plasma from rats and humans. VEA is an isomer of cis-oleic acid ethanolamide (OEA). OEA, palmitic acid ethanolamide (PAE) and stearic acid ethanolamide (SEA) were found to occur physiologically in several biological samples in addition to the endocannabinoid arachidonic acid ethanolamide anandamide (AEA; reviewed in Ref. [2]). OEA, VEA, PEA, and SEA are not endocannabinoids. Previously, we observed by GC-MS/MS in the selected-reaction monitoring (SRM) mode two chromatographic peaks with very similar retention times. We identified the compound eluting at $17.86 \mathrm{~min}$ as OEA, yet the peak eluting at 17.92 remained unknown [3]. The findings by Röhrig et al. [1] suggest that the unknown peak we observed in our study [3] is likely to be VEA. Interestingly, the mean peak area ratio of OEA to VEA in plasma samples from our group of about 3.5 is close to the mean value of 2.4 reported by Röhrig et al. for human plasma samples [1]. In plasma of 16 apparently healthy humans, we measured by GC-MS/MS OEA concentrations of $18 \pm 6 \mathrm{nM}$, which suggest an approximate VEA mean concentration of $5 \mathrm{nM}$. Even this concentration is higher than the plasma AEA concentration of $1.4 \mathrm{nM}$ [3]. Previously, we demonstrated that a considerable fraction of

Dimitrios Tsikas

tsikas.dimitros@mh-hannover.de

1 Centre of Pharmacology and Toxicology, Hannover Medical School, Carl-Neuberg-Str. 1, 30625 Hannover, Germany plasma OEA but not of AEA is due to laboratory contamination [4]. Yet, we did not investigate whether the putative VEA may also originate from laboratory contamination or from OEA derivatization and GC-MS/MS analysis, which require must more drastic conditions compared with UPLC-MS/MS.

Reply by the authors of [1]:

We thank Dr. Dimitrios Tsikas for this highly interesting comment, which provides an important link between our study and Dr. Tsikas' own excellent contributions in this field.

\section{Compliance with Ethical Standards}

Conflict of interest The author declares that there is no conflict of interest.

\section{References}

1. Röhrig W, Waibel R, Perlwitz C, Pischetsrieder M, Hoch T. Identification of the oleic acid ethanolamide (OEA) isomer cisvaccenic acid ethanolamide (VEA) as a highly abundant 18:1 fatty acid ethanolamide in blood plasma from rats and humans. Anal Bioanal Chem. 2016;408(22):6141-51.

2. Zoerner AA, Gutzki FM, Batkai S, May M, Rakers C, Engeli S, et al. Quantification of endocannabinoids in biological systems by chromatography and mass spectrometry: a comprehensive review from an analytical and biological perspective. Biochim Biophys Acta. 2011;1811(11):706-23.

3. Zoerner AA, Gutzki FM, Suchy MT, Beckmann B, Engeli S, Jordan J, et al. Targeted stable-isotope dilution GC-MS/MS analysis of the endocannabinoid anandamide and other fatty acid ethanol amides in human plasma. J Chromatogr B. 2009;877(26):2909-23.

4. Tsikas D. Identifying and quantifying contaminants contributing to endogenous analytes in gas chromatography/mass spectrometry. Anal Chem. 2010;82(18):7835-41. 